\title{
Trajectory of the August 7, 2010 Biwako fireball determined from seismic recordings
}

\author{
Masumi Yamada and Jim Mori \\ Disaster Prevention Research Institute, Kyoto University, Kyoto, Japan \\ (Received January 27, 2011; Revised August 31, 2011; Accepted August 31, 2011; Online published March 2, 2012)
}

\begin{abstract}
The Biwako reball on August 7, 2010, produced a strong sonic boom throughout central Japan around 17:00 JST (UTC+9). There were many visual observations and reports of the sound in the Tokai and Kinki regions at that time. We have estimated the trajectory of this reball and the location of its termination point by analyzing seismograms recorded on a dense local network. The isochrons of the arrival times are close to concentric circles, which suggest that the reball disappeared due to fragmentation during entry. The reball trajectory which explains the arrival times of the signal has a relatively high incident angle (55 degrees relative to the horizon) and the source is thought to disappear at a height of 26-km east of Lake Biwa. The azimuthal angle and velocity of the reball are dif cult to determine uniquely from this dataset. We identi ed an event thought to be due to fragmentation, with a location 3-km ENE and 9-km higher than the termination point. This location is consistent with the trajectory determined from the signal arrival. Based on this trajectory model, the source of the signal spans a horizontal range of 26 to $70 \mathrm{~km}$, and the altitude of the source producing the sonic boom is about 30 to $50 \mathrm{~km}$.
\end{abstract}

Key words: 2010 Biwako reball, estimation of trajectory, sonic boom, meteoroid, fragmentation.

\section{Introduction}

The Lake Biwa (Biwako in Japanese) reball on August 7, 2010, produced a strong sonic boom throughout central Japan at around 17:00 JST (UTC+9). There were numerous reports of the track across the sky and associated sounds in the region. A newspaper reported that the sonic boom was heard by local residents in the Tokai region (Aichi, Gifu, and Mie prefectures) and some people called emergency services to report the strong sound (asahi.com, 2010). Bright ashes were observed as far away as $250 \mathrm{~km}$ from the termination point, as reported on an internet bulletin board (Japan Fireball Network, 1999). Local amateur astronomers have searched for the meteoroid which may have reached the ground, but nothing has been found so far (Onishi, 2010).

Past atmospheric trajectories of reballs have been determined by visual recordings such as photographs and movies (Brown et al., 1994, 2003), infrasound records (Brown et al., 2002; Le Pichon et al., 2002, 2008), and seismic records (e.g., Nagasawa, 1978; Nagasawa and Miura, 1987; Cevolani, 1994; Qamar, 1995; Brown et al., 2002; Cates and Sturtevant, 2002; Le Pichon et al., 2002, 2008; Ishihara et al., 2003, 2004; Rydelek and Pujol, 2004; Pujol et al., 2005). An object ying at supersonic velocity produces a sonic boom, and the acoustic-to-seismic coupled signal is often recorded by seismic arrays. The airwave signal from this reball was recorded by about 50 seismic stations in

Copyright (c) The Society of Geomagnetism and Earth, Planetary and Space Sciences (SGEPSS); The Seismological Society of Japan; The Volcanological Society of Japan; The Geodetic Society of Japan; The Japanese Society for Planetary Sciences; TERRAPUB.

doi:10.5047/eps.2011.08.021
Japan (NIED, 2010). This is one of the few events having an airwave signal that has been recorded by a dense seismic network (Walker et al., 2010). In this paper, we estimate the trajectory of the Biwako reball and the location of the termination point of the signal by analyzing the seismograms, and discuss the characteristic waveforms and mechanism of reball fragmentation.

\section{Data}

\subsection{Seismic data}

The Biwako reball produced atmospheric sound waves that were recorded by Hi-net, F-net (both operated by the National Research Institute for Earth Science and Disaster Prevention), the Japan Meteorological Agency highsensitivity seismic network, and high-sensitivity seismic networks operated by Japanese universities (Okada et al., 2004). Those seismic networks currently have about 20$\mathrm{km}$ spacing throughout Japan, and we identi ed shockwave signals from the reball at 49 stations.

Since the onsets of the signal arrival are not very clear, we determined the arrival times as follows. First, a bandpass Butterworth lter with typical cutoff frequencies of 2 to $8 \mathrm{~Hz}$ was applied to each record to look at the frequency range of the infrasound signals. Since we used records from various types of sensors, we tried alternative high-pass Butterworth lters if the signal was not clear. The ltered records are shown in Fig. 1. We used only vertical components for this analysis. The onset of the signal is determined by tting a multi-variate locally-stationary autoregressive (MLSAR) model (Takanami and Kitagawa, 1991). This technique is widely used to select $P$-wave arrivals in the seismograms. The characteristics of the time series change 


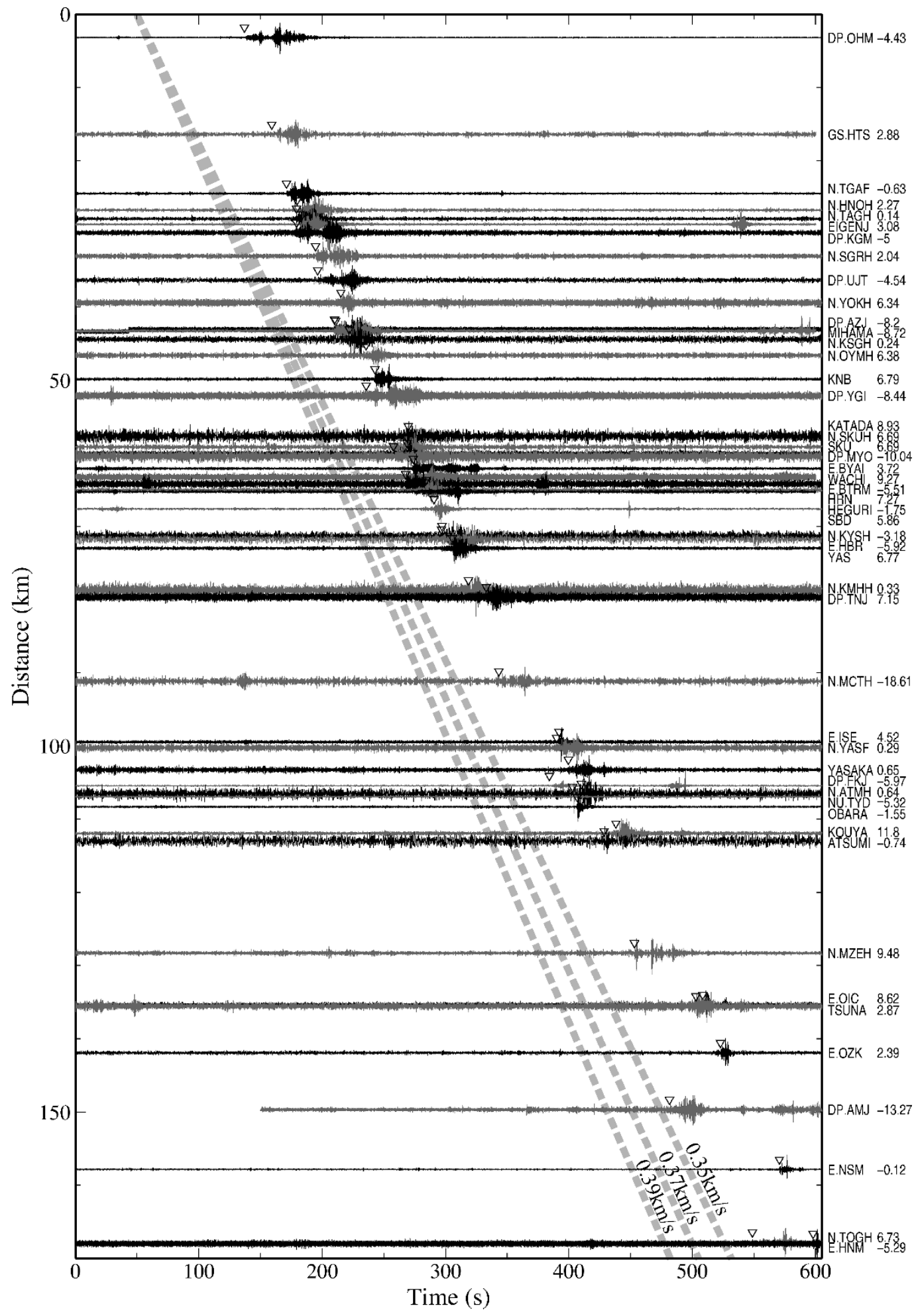

Fig. 1. Waveforms ordered as a function of distance from the termination point. The open triangles show the observed arrival times. The station code and residual of the arrival time $\left(t_{\text {pred }}-t_{\mathrm{obs}}\right)$ are added on the right side. Alternate seismograms are shown in black and gray for clarity.

over time due to the arrival of seismic waves. Therefore, the time series is divided into two segments, and the autoregressive model is tted to each segment. The log-likelihood of each multi-variate locally-stationary autoregressive model is computed, and the Akaike information criterion (AIC) is used to determine the best onset time (Akaike, 1974). The onset of the signal is determined as the section that min- imizes the AIC. Although this technique can identify the time that the characteristics of the waveforms show the most signi cant changes, there is a possibility that this onset time is contaminated by an air-coupled Rayleigh wave (Edwards et al., 2008). The arrival times determined by this method are marked as open triangles in Fig. 1.

In contrast to some past observations (e.g., Cates and 


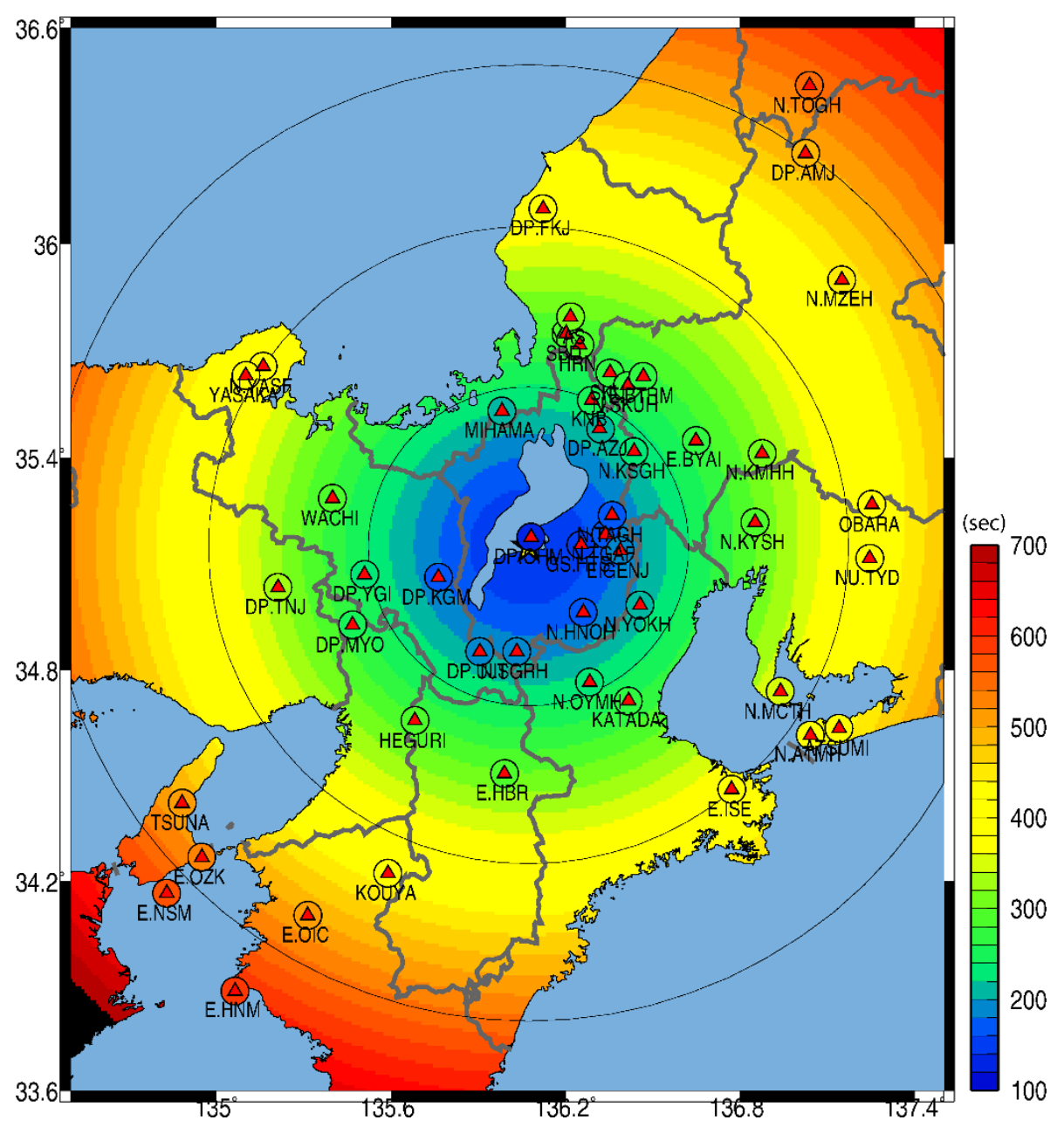

Fig. 2. Observed arrival times of the shockwave (color of the small circle at stations) and predicted arrival times of the shockwave based on the trajectory model (color contors). The large circles indicate distances of 50,100, and $150 \mathrm{~km}$ from the estimated termination point of the signal.

Sturtevant, 2002; Ishihara et al., 2003), the airwave signals from this event have unclear emergent onsets, a long duration (10-40 s), and no distinct " $\mathrm{N}$ " shaped waves. The low apparent velocity $(\sim 0.37 \mathrm{~km} / \mathrm{s})$ of the signals across the seismograph network indicates that the source of the signal is in the atmosphere, and is not an earthquake (see Fig. 1).

Figure 2 shows the distribution of the arrival times of the shockwave. The first arrival was recorded at station DP.OHM (Ohmi-Hachiman) east of Lake Biwa, which indicates that the termination of the fireball is close to this station. The area where the signals are observed is within $170 \mathrm{~km}$ from this station. A notable feature of this fireball is that the isochron pattern consists of nearly concentric circles, not half-ellipses, as observed in most past studies (Cates and Sturtevant, 2002; Ishihara et al., 2003).

\subsection{Visual observations}

The Biwako fireball was observed by many people, even in daylight, around 17:00 local time, early evening in the summer. Witnesses reported the locations and times of sightings, the durations of the flash, and the directions of the fireball emergence, on an internet bulletin board (Japan Fireball Network, 1999). Figure 3 shows the direction of the fireball observed by 34 witnesses. The fireball was observed from sites more than $250 \mathrm{~km}$ away from the fireball trajectory. Although their reports are relatively rough (16point compass directions, such as WSW, are usually used), the direction of the fireball is consistent in all reports. We can estimate that the explosive signal was produced near Lake Biwa from this figure. Some reports describe a fireball traveling with bright illumination twice to three times the size of the moon, and splitting into several parts before disappearing. Most of the witnesses reported that the white path of the meteor remained for a few minutes in the sky. The diamond symbols in Fig. 3 represent the sites where sonic booms were observed (asahi.com, 2010). Here, a sonic boom is defined as a strong explosive sound with an impact, so sounds resembling distant thunder are excluded. Compared to the visual observations of the fireball, the observations of the sonic boom were in a more limited area.

\section{Methods}

We estimated the trajectory of the fireball and the termination point of the signal using a method similar to Nagasawa and Miura (1987). This method assumes straight-ray theory and a constant velocity of sound. Since the arrival pattern of the signals are close to concentric circles, we assume that the fireball travelled toward the earth and terminated in the air (or, at least, stopped producing 


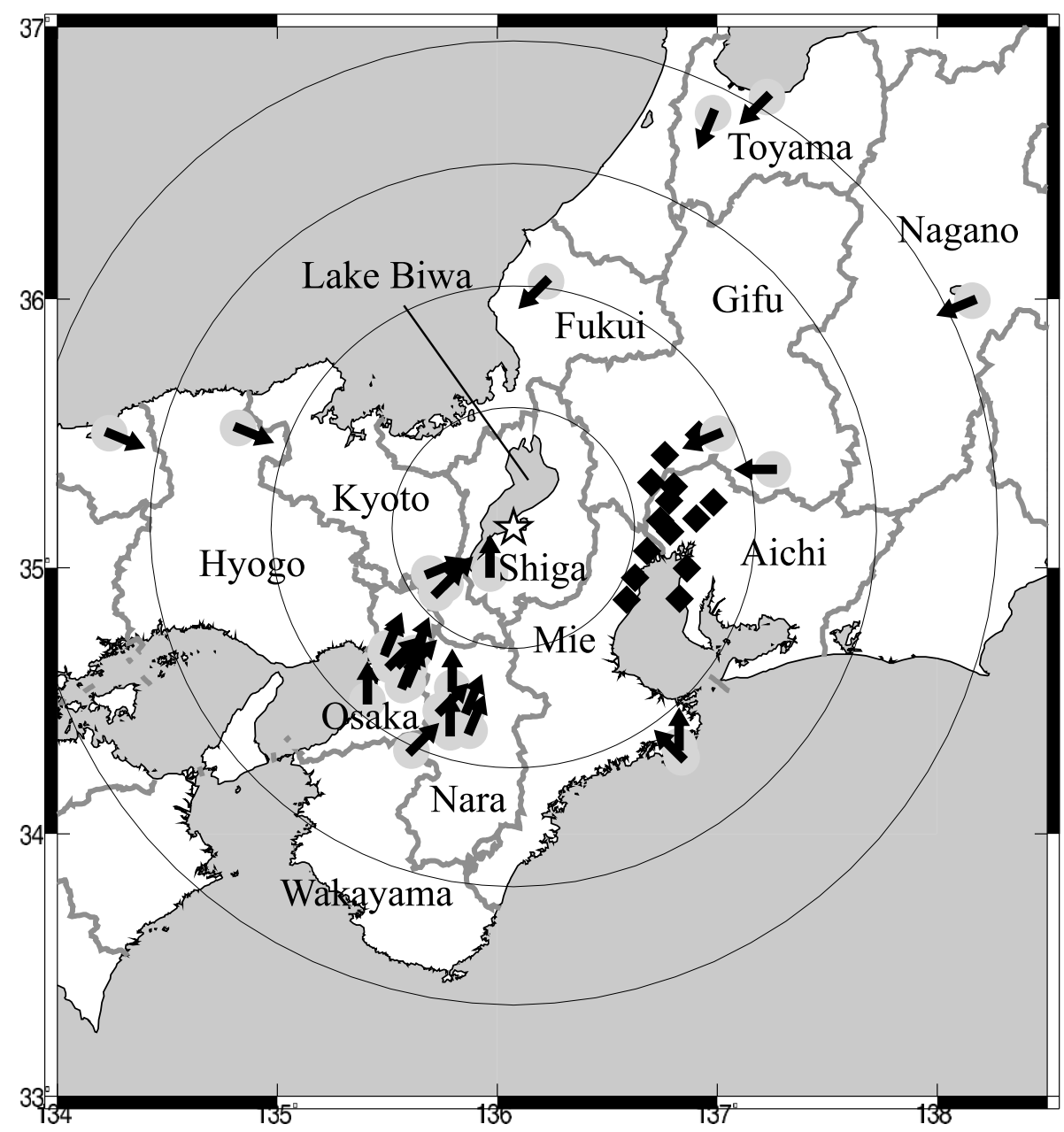

Fig. 3. Directions of the fireball observed by 34 witnesses. The diamond symbols show the sites where sonic booms were heard. Star shows the termination point of the signal. The large circles show distances of 50,100,150, and $200 \mathrm{~km}$ from the estimated termination point of the signal.

atmospheric signals). A combination of line and point sources is considered to have produced this arrival pattern. First, the fireball moved through the air with a high velocity, producing nearly cylindrical ballistic waves with an elliptical arrival pattern on the ground. Then, the fireball experienced a catastrophic fragmentation, or disruption, at a termination point, which caused arrivals in a pattern of concentric circles (Edwards et al., 2008). The sites on the ground in the direction of the fireball trajectory record signals due to the fragmentation, while sites on the ground perpendicular to the fireball trajectory record the ballistic wave and signals from the fragmentation (see Fig. 4). Based on this assumption, we estimated the trajectory and termination point of the signal.

The estimated arrival times of the shockwave are represented with the following function (Nagasawa and Miura, 1987). Here, we have added an assumption that the object dissipates and that the generation of the shock wave stops at the termination point of the fireball, so extra parameters are added to the function.

$$
\begin{aligned}
& t_{\text {pred }}=t_{0}+1 / v\left(\sqrt{X^{2}+Y^{2}} / \tan \beta-Z\right) \\
& t_{\text {pred }}=t_{0}+1 / c\left(\sqrt{X^{2}+Y^{2}+Z^{2}}\right)
\end{aligned}
$$

$$
\text { if }\left\{\begin{aligned}
Z \geq & -\tan \beta \sqrt{X^{2}+Y^{2}}, \\
& \text { Eq. (1) (region A in Fig. 4) } \\
Z<- & \tan \beta \sqrt{X^{2}+Y^{2}}, \\
& \text { Eq. (2) (region B in Fig. 4), }
\end{aligned}\right.
$$

where,

$$
\beta=\arcsin (c / v)
$$

$$
\left(\begin{array}{l}
X \\
Y \\
Z
\end{array}\right)=\left(\begin{array}{ccc}
-\sin \gamma & \cos \gamma & 0 \\
-\cos \gamma \sin \theta & -\sin \gamma \sin \theta & \cos \theta \\
\cos \gamma \cos \theta & \sin \gamma \sin \theta & \sin \theta
\end{array}\right)\left(\begin{array}{l}
x-x_{0} \\
y-y_{0} \\
z-z_{0}
\end{array}\right)
$$

$x_{0}, y_{0}, z_{0}$ : coordinates of the trajectory where the signal vanished

$t_{0}:$ time when the signal vanished

$\gamma:$ azimuth of the trajectory

$\theta$ : incident angle of the trajectory relative to the horizontal

$c$ : velocity of sound $(0.312 \mathrm{~km} / \mathrm{s})$

$v$ : velocity of the fireball $(20 \mathrm{~km} / \mathrm{s})$

The coordinate system used in this analysis is shown in Fig. 4. The origin for the $x, y, z$ coordinate system is defined as longitude $136^{\circ} \mathrm{E}$, latitude $35^{\circ} \mathrm{N}$, and altitude $0 \mathrm{~km}$, and the origin time is 17:00:00 JST. The speed of the 


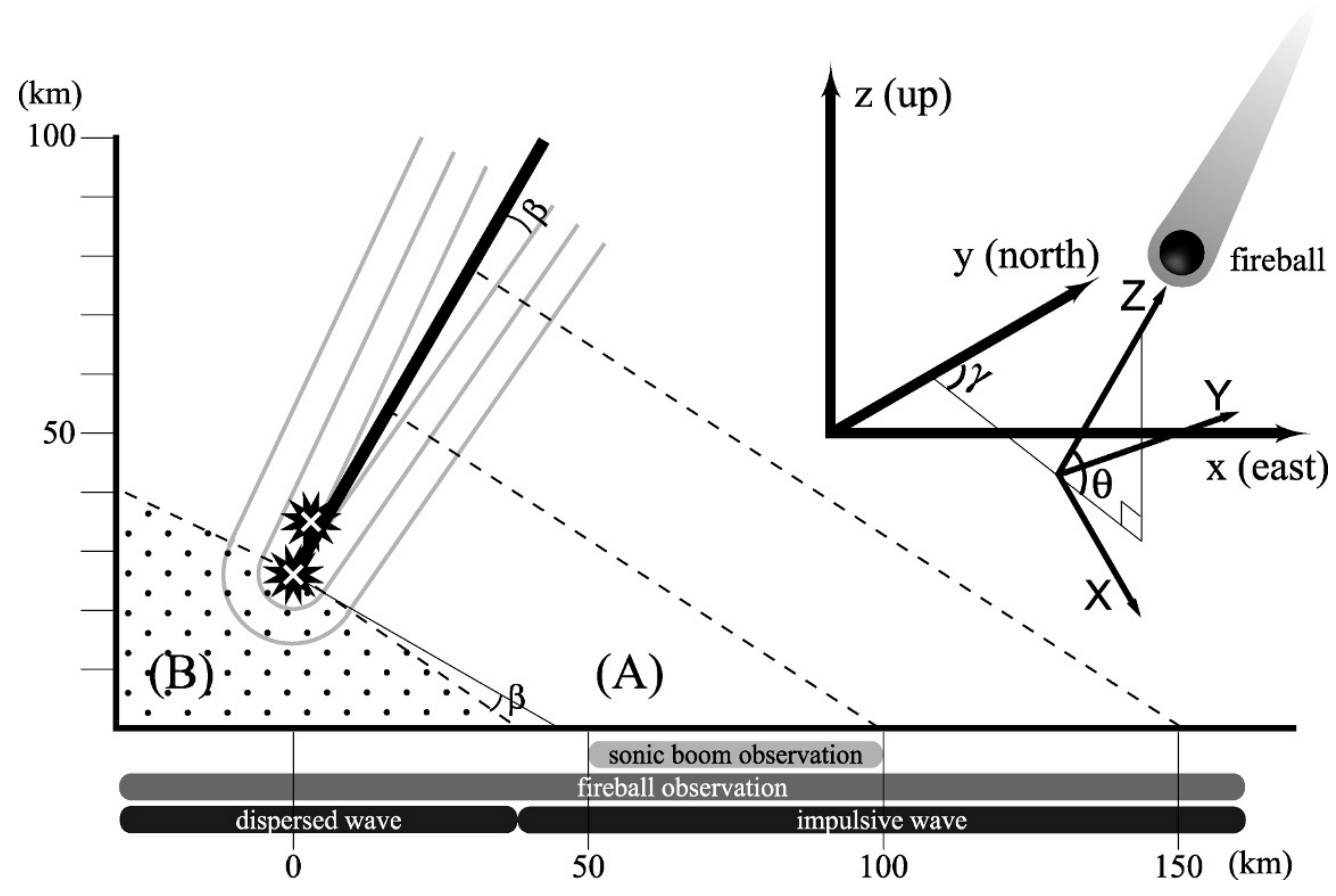

Fig. 4. Schematic diagram of meteor-generated atmospheric waves. In region A, the signal is explained by a line souce, and in region B by a point source. Coordinate systems used in the analysis are shown on the right-hand side. Origins for the $x, y, z$ coordinate system are longitude 136E, latitude $35 \mathrm{~N}$, and $0 \mathrm{~km}$ altitude, respectively. See the text for details.

sonic wave is assumed to be a constant with the value of $0.312 \mathrm{~km} / \mathrm{s}$ taken from Nagasawa and Miura (1987). The velocity of the reball is a parameter which is dif cult to constrain because of the trade off with the time the signal vanishes, since the velocity of sound is slow with respect to the observed duration of the signal assuming a range of reasonable reball velocities (Ishihara et al., 2003; Edwards et al., 2008). We computed a mis $\mathrm{t}$ surface for each parameter (see Fig. 5) and the result shows that the velocity of the reball is not sensitive to the root-mean-square residual of the arrival times, so a xed velocity of $20 \mathrm{~km} / \mathrm{s}$ was used. (The mis $\mathrm{t}$ surface will be discussed in the next section.) Six free parameters $\left(x_{0}, y_{0}, z_{0}, t_{0}, \gamma, \theta\right)$ that de ne the reball trajectory are solved by minimizing the rms residuals:

$$
\mathrm{rms}=\sqrt{\frac{1}{n-6} \sum_{i=1}^{n}\left(t_{\mathrm{pred}, i}-t_{\mathrm{obs}, i}\right)^{2}},
$$

where $n$ is the number of observations, $t_{\mathrm{pred}, i}$ is the predicted arrival time of the signal at the $i$ th station, and $t_{\mathrm{obs}, i}$ is the measured arrival time of the signal at the $i$ th station. The 6 in the denominator is the number of parameters to be estimated (Montgomery and Runger, 2003).

\section{Analysis and Results}

\subsection{Estimation of the trajectory}

We performed a grid search to nd the most probable set of parameters of the trajectory model. The best- tting parameters of the trajectory that explain the arrival times of the shock wave are shown in Table 1. Intervals of the grid search, search ranges, and con dence intervals of the parameters are also included in the table. The isochrons of the arrival times based on this model are shown in Fig. 2, and the residuals of the arrival times are shown in Fig. 1. The location of the termination point of the signal is east of Lake Biwa, and at an altitude of $26 \mathrm{~km}$. The $95 \%$ con dence interval of each parameter is calculated by a bootstrap method with 100 replicates (Efron and Tibshirani, 1993; Walker et al., 2010). The uncertainties of the parameters $x_{0}, y_{0}, z_{0}, t_{0}, \theta$ are small, since the arrival time of the shock wave is sensitive to these parameters. However, the azimuthal angle $\gamma$ has a large con dence interval and is difcult to determine uniquely from this dataset, since most of the stations are inside the concentric isochrons and the azimuthal coverage of stations with distances greater than $100 \mathrm{~km}$ is poor.

\subsection{Sensitivity analysis}

We computed mis $t$ surfaces as a function of each parameter to check the sensitivity of the parameters. The minimum of the rms residuals are computed as a function of two selected parameters (see Fig. 5). The mis $t$ surface for the horizontal location is smooth in both longitude and latitude, and has a single local minimum. Therefore, the solution easily converges to this minimum. The optimal time and altitude of the meteorite dissipation are both sensitive to the velocity of sound and dif cult to resolve, but, nevertheless, a broad minimum exists in the surface. Note that these two parameters are also sensitive to the sound velocity. The perturbation of the parameters is about $10 \%$ if we change the velocity of the sound by $0.01 \mathrm{~km} / \mathrm{s}$. The azimuth of the trajectory is not very well determined by the dataset, as we have seen with the con dence interval. The velocity of the reball is also signi cantly insensitive to the data, so we used a constant velocity for this analysis. 

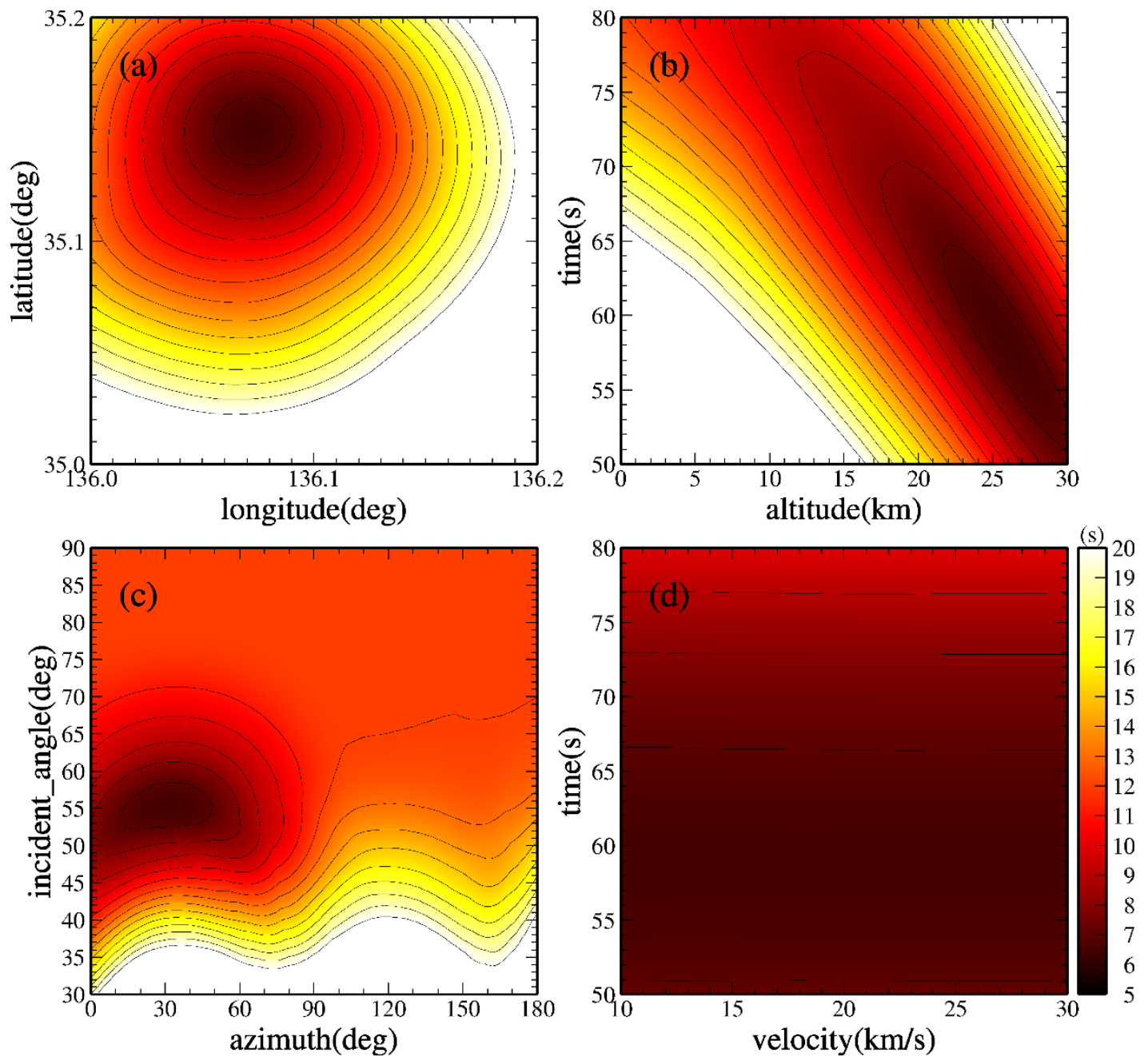

Fig. 5. Misfit surface for parameters. The rms residuals are computed as a function of two out of six of the parameters to see the trade-off between parameters.

Table 1. The most probable parameters which determine the trajectory of the fireball.

\begin{tabular}{l|cccc}
\hline \multicolumn{1}{c|}{ Parameters } & Optimal solution & Search range & Grid interval & Confidence interval \\
\hline Longitude (deg.) & 136.073 & $136.0-136.2$ & 0.001 & $136.055-136.090$ \\
Latitude (deg.) & 36.149 & $35.0-35.2$ & 0.001 & $35.140-35.160$ \\
Height (km) & 26 & $0-50$ & 1 & $22-30$ \\
Time (sec) & 58 & $50-80$ & 1 & $53-65$ \\
Incident ang. (deg.) & 55 & $30-90$ & 1 & $51-60$ \\
Azimuth (deg.) & 32 & $0-180$ & 1 & $16-63$ \\
\hline RMS (sec) & 6.86 & - & - & - \\
\hline
\end{tabular}

\subsection{Interpretation of the model}

A mechanism to produce the concentric isochrons of the arrival times can be explained by an explosive fragmentation (Edwards et al., 2008). During a meteoroid entry, the object breaks up suddenly because of the increasingly large air pressure. A large amount of light is produced associated with the break up. Since these explosive fragmentation events are very brief and take place over small portions of the entire trajectory, they are approximated by a point source, and result in the concentric isochrons.

The non pulse-like waveforms can also be explained with this mechanism. The fragmentation may result in the sep- aration of the original body into several large fragments. The duration of the fragmentation is largely unknown; however, if fragmentation takes 0.5 second, the meteoroid can travel as far as $10 \mathrm{~km}$ (assuming a constant velocity) during this time. This distance is comparable to a difference of 32 seconds in the arrival time of the waveforms. Therefore, the extremely long duration of the signal is not necessarily unreasonable (Walker et al., 2010). Edwards et al. (2008) explained that observations of explosive pointsource events tend to be diffuse, with no distinct arrival time, in contrast to the sharp onset of ballistic observations. Waveforms here are very similar to the waveforms of the 
Table 2. The most probable parameters which determine the trajectory of the reball from a point source model.

\begin{tabular}{l|ccc}
\hline \multicolumn{1}{c|}{ Parameters } & Optimal solution & Search range & Grid interval \\
\hline Longitude (deg.) & 136.086 & $136.0-136.2$ & 0.001 \\
Latitude (deg.) & 36.172 & $35.0-35.2$ & 0.001 \\
Height (km) & 33 & $0-50$ & 1 \\
Time (sec) & 45 & $40-70$ & 1 \\
\hline RMS (sec) & 12.07 & - & - \\
\hline
\end{tabular}

Table 3. The most probable parameters which determine the trajectory of the reball from a back projection.

\begin{tabular}{l|cccccc}
\hline \multicolumn{1}{c|}{ Parameters } & $55 \mathrm{sec}$ & $56 \mathrm{sec}$ & $57 \mathrm{sec}$ & $58 \mathrm{sec}$ & $59 \mathrm{sec}$ & $60 \mathrm{sec}$ \\
\hline Longitude (deg.) & 136.102 & 136.091 & 136.108 & 136.104 & 136.109 & 136.115 \\
Latitude (deg.) & 35.169 & 35.168 & 35.162 & 35.168 & 35.163 & 35.163 \\
Height (km) & 36 & 35 & 36 & 34 & 35 & 35 \\
\hline$Q_{t}$ & 0.521 & 0.537 & 0.533 & 0.515 & 0.533 & 0.519 \\
\hline
\end{tabular}

2002 Tagish Lake reball (Brown et al., 2002) and 1989 St. Helens reball (Qamar, 1995), which both show concentric isochrons. Similar concentric isochrones were also observed in Arrowsmith et al. (2007) and Walker et al. (2010). Multipathing through the atmosphere might also complicate the character of the waveforms; however, this is generally observed at distances greater than $200 \mathrm{~km}$ (Walker et al., 2010), which is not the range of the data in this study.

\subsection{Height of the source}

The airwave signal was observed by seismometers as far away as $150 \mathrm{~km}$, and bright ashes were observed as far away as $250 \mathrm{~km}$ from the termination point (Japan Fireball Network, 1999). We have tried to estimate the altitude of the termination point from the trajectory model. Figure 4 shows a schematic diagram of the reball trajectory and meteor-generated atmospheric waves. If the source dissipates at $26-\mathrm{km}$ altitude, the ballistic wave due to the object ying at supersonic speed cannot be observed within $40 \mathrm{~km}$ from the epicenter. The signal observed in this near-source region is not impulsive due to the fragmentation. Assuming a line source, the height of a source which is observable $150 \mathrm{~km}$ from the center of the arrival time pattern must be at least $70-\mathrm{km}$ high. Therefore, the source of the signal is inferred to be between 26 and $70 \mathrm{~km}$, and could be higher if there is strong attenuation in the atmosphere. Reports of the sonic boom concentrate in the Tokai region, about $50-100 \mathrm{~km}$ from the center of the arrival-time pattern. The altitude of the source corresponding to this signal is about 30- to $50-\mathrm{km}$ high. This height is consistent with past observations; 22 to $34 \mathrm{~km}$ for the 2000 Moravka reball and 34 to $87 \mathrm{~km}$ for the 2003 Kanto reball (Pujol et al., 2006). For the Biwako reball, we located two fragmentation events at heights of 26 and $35 \mathrm{~km}$ (see Tables 1 and 3). It has been suggested that the height of fragmentation is where the aerodynamic pressure exceeds the material strength. Cevolani (1994) calculated the critical heights of the rst fragmentation for meteoroids having different values of material strength. According to these results, the critical height is 45.5 to $56.5 \mathrm{~km}$ for dustballs, 14 to $38 \mathrm{~km}$ for stony chondrites, and 3 to $14 \mathrm{~km}$ for metal bodies, at velocities of 15 to $30 \mathrm{~km} / \mathrm{s}$. From these values, we speculate that the material of the Biwako reball may be stony chondrite.

\section{Different Models}

\subsection{Point source model}

We also examined a simple point source model to try to explain the same dataset. If the observed arrivals are produced by a terminal explosion in a very short time frame, the arrival-time pattern should be explained by a simple point source model. We used the same dataset as in Section 2, and the same method as in Section 3, except that the velocity of the reball, and the incident angle and azimuth of the trajectory, are now set to be zero. The estimated arrival times are computed from Eq. (1). The most probable parameter set from the grid search is shown in Table 2. We compared the two models (point source plus line source model, and simple point source model) with an F-test. The null hypothesis is the case where the two models predict the dataset equally well. The F statistic is given by

$$
\mathrm{F}=\left(\frac{\mathrm{RSS}_{1}-\mathrm{RSS}_{2}}{p_{2}-p_{1}}\right) /\left(\frac{\mathrm{RSS}_{2}}{n-p_{2}}\right)=48.11
$$

where $\mathrm{RSS}_{i}$ is the residual sum of squares of model $i, p_{i}$ is the number of parameters of model $i$, and $n$ is the number of observations. From the $\mathrm{F}$ distribution table, the $\mathrm{F}$ value with $\left(p_{2}-p_{1}, n-p_{2}\right)$ degrees of freedom at a $5 \%$ signi cance level is 3.21. Therefore, the null hypothesis has a low probability of being accepted, and the more complicated model provides a signi cantly better $t$ to the data.

\subsection{Back projection method}

Since we used the times of the onset of the signal for the location estimation, these arrival times correspond to the location of the end of the terminal explosion. We applied a back projection method to the waveforms in order to nd the location where the reball produced the largest energy.

The waveforms used here are the same as the dataset in Section 2. Since the correlation distance of infrasound at 0.5 to $5 \mathrm{~Hz}$ is only several kilometers (Walker et al., 2010), we used the envelopes of the waveforms. The data are processed as follows; envelopes of the waveforms are formed using the maximum absolute value of the waveforms over one-second windows. To remove the effect of stationary noise, the mean over a 10 -minute duration is removed. Then, the maximum amplitude of the signal is nor- 


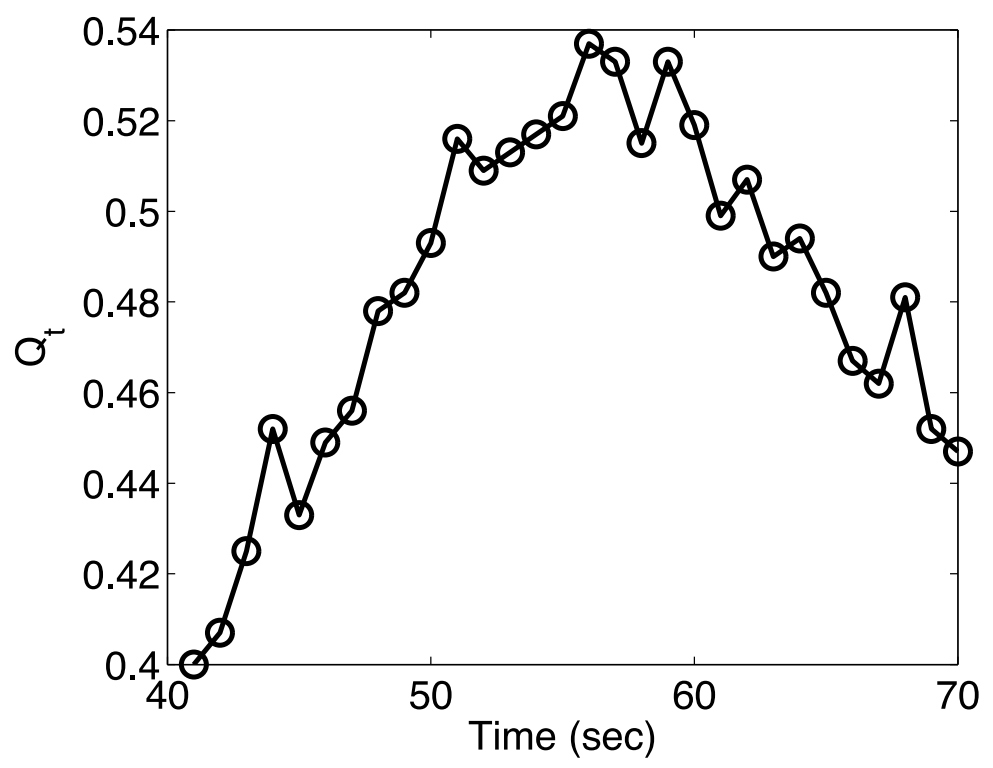

Fig. 6. Amplitudes of the stack of the waveforms from the back projection analysis, as a function of time.

malized to one to regularize the amplitude of the envelopes.

The back projection method used here is similar to the reverse time migration technique in Walker et al. (2010). However, the stack of the waveform amplitudes $(Q)$ is defined as a function of longitude, latitude, altitude, and time, in our analysis. The maximum for every second $t$ is defined as $Q_{t}$. The weighting is set to be one since our station distribution is not greatly skewed. The search range is the same as shown in Table 2. Figure 6 shows $Q_{t}$ as a function of $t$. The $Q_{t}$ has a local maxima between 55 and 60 seconds, and the optimal parameters at those times are shown in Table 3.

The computed location of the source producing the largest energy is located around $136.105 \mathrm{E}$ and $36.165 \mathrm{~N}$, about $3-\mathrm{km}$ ENE and $9-\mathrm{km}$ higher than the termination point. This location is consistent with the trajectory determined from the arrivals at the seismic stations, since the path from the source producing the largest energy to the terminal point is similar to the calculated trajectory. Because the source of the largest energy is at a higher altitude than the termination burst, it was recorded a few tens of seconds later at some stations.

\section{Conclusions}

We have estimated the trajectory of the August 7, 2010, Biwako fireball, and the location of its termination point from arrivals at seismic stations. The isochrons of the arrival times are nearly concentric circles, which suggest that the fireball dissipated due to fragmentation during entry. The fireball trajectory which explains the arrival times of the signal has a relatively high incident angle (55 degrees) and the source is thought to disappear at a height of $26-\mathrm{km}$ east of Lake Biwa. The azimuthal angle and velocity of the fireball are difficult to determine uniquely from this dataset. We identified an event thought to be due to fragmentation, with a location 3-km ENE and 9-km higher than the termination point. This location is consistent with the trajectory determined from the arrival time data. Based on this trajec- tory model, the location of the source of the signal spans a range of 26 to $70 \mathrm{~km}$, and the altitude of the source producing the sonic boom is about 30 to $50 \mathrm{~km}$.

Acknowledgments. The authors thank Yoshihisa Iio of Kyoto University for providing the data observed west of Lake Biwa. We acknowledge the National Research Institute for Earth Science and Disaster Prevention (NIED) and the Japan Meteorological Agency (JMA) for the use of the seismic data. This research was supported by the Program for Improvement of Research Environment for Young Researchers from Special Coordination Funds for Promoting Science and Technology (SCF) commissioned by the Ministry of Education, Culture, Sports, Science and Technology (MEXT) of Japan.

\section{References}

Akaike, H., A new look at the statistical model identification, IEEE Trans. Automat. Contr., 19, 716-723, 1974.

Arrowsmith, S., D. Drob, M. Hedlin, and W. Edwards, A joint seismic and acoustic study of the Washington state bolide: Observations and modeling, J. Geophys. Res., 112, D09304, 2007.

asahi.com, Reported detonation in the wide area in Tokai region, no damage reported, http://www.asahi.com/special/playback/ ngy201008070006.html, accessed 20 November 2010, 2010 (in Japanese).

Brown, P., Z. Ceplecha, R. Hawkes, G. Wetherill, M. Beech, and K. Mossman, The orbit and atmospheric trajectory of the Peekskill meteorite from video records, Nature, 367, 624-625, 1994.

Brown, P., D. Revelle, E. Tagliaferri, and A. Hildebrand, An entry model for the Tagish Lake fireball using seismic, satellite and infrasound records, Meteorit. Planet. Sci., 37, 661-676, 2002.

Brown, P., P. Kalenda, D. Revelle, and J. Borovicka, The Moravka meteorite fall: 2 . interpretation of infrasonic and seismic data, Meteorit. Planet. Sci., 38, 989-1003, 2003.

Cates, J. and B. Sturtevant, Seismic detection of sonic booms, J. Acoust. Soc. Am., 111, 614-628, 2002.

Cevolani, G., The explosion of the bolide over Lugo di Romagna (Italy) on 19 January 1993, Planet. Space Sci., 42, 767-775, 1994.

Edwards, W., D. Eaton, and P. Brown, Seismic observations of meteors: Coupling theory and observations, Rev. Geophys., 46, RG4007, 2008.

Efron, B. and R. Tibshirani, An Introduction to the Bootstrap, Chapman \& Hall, 1993.

Ishihara, Y., S. Tsukada, S. Sakai, Y. Hiramatsu, and M. Furumoto, The 1998 Miyako fireball's trajectory determined from shock wave records of a dense seismic array, Earth Planets Space, 55, 9-12, 2003. 
Ishihara, Y., M. Furumoto, and S. Sakai, The 2003 Kanto large bolide's trajectory determined from shockwaves recorded by a seismic network and images taken by a video camera, Geophys. Res. Lett., 31, L14702, 2004.

Japan Fireball Network, Bulletin board of the Japan reball network, http://www3.cnet.ne.jp/c-shimo/index.html, accessed 20 November 2010, 1999 (in Japanese).

Le Pichon, A., J. Guérin, E. Blanc, and D. Reymond, Trail in the atmosphere of the 29 December 2000 meteor as recorded in Tahiti: Characteristics and trajectory reconstitution, J. Geophys. Res., 107, 4709, 2002.

Le Pichon, A., K. Antier, Y. Cansi, B. Hernandez, E. Minaya, B. Burgoa, D. Drob, L. Evers, and J. Vaubaillon, Evidence for a meteoritic origin of the September 15, 2007, Carancas crater, Meteorit. Planet. Sci., 43, 1797-1809, 2008.

Montgomery, D. and G. Runger, Applied Statistics and Probability for Engineers, 3rd edition, Wiley and Sons, 2003.

Nagasawa, K., An analysis of sonic boom from a great reball on May 10 1977, recorded on seismographs of volcano observatories, Bull. Earthq Res. Inst. Univ. Tokyo, 53, 271-280, 1978.

Nagasawa, K. and K. Miura, Aerial path determination of a great reball from sonic boom records on seismographs, Bull. Earthq. Res. Inst. Univ. Tokyo, 62, 579-588, 1987.

NIED, The signal from the August 7, 2010 reball observed by Hinet, NIED, http://www.hinet.bosai.go.jp/topics/fball100807/, accessed 20 November 2010, 2010 (in Japanese).

Okada, Y., K. Kasahara, S. Hori, K. Obara, S. Sekiguchi, H. Fujiwara, and A. Yamamoto, Recent progress of seismic observation networks in Japan-Hi-net, F-net, K-NET and KiK-net, Earth Planets Space, 56, 15-28, 2004.

Onishi, M., Search for a meteor which might have reached around $\mathrm{Bi}$ wako, Kyoto Shimbun, November 7, 2010, local news section, 2010 (in Japanese).

Pujol, J., P. Rydelek, and T. Bohlen, Determination of the trajectory of a reball using seismic network data, Bull. Seismol. Soc. Am., 95, 14951509, 2005.

Pujol, J., P. Rydelek, and Y. Ishihara, Analytical and graphical determination of the trajectory of a reball using seismic data, Planet. Space Sci., 54, 78-86, 2006.

Qamar, A., Space shuttle and meteoroid-tracking supersonic objects in the atmosphere with seismographs, Seismol. Res. Lett., 66, 6-12, 1995.

Rydelek, P. and J. Pujol, Real-time seismic warning with a two-station subarray, Bull. Seismol. Soc. Am., 94, 1546-1550, 2004.

Takanami, T. and G. Kitagawa, Estimation of the arrival times of seismic waves by multivariate time series model, Ann. Inst. Stat. Math., 43, 407433, 1991.

Walker, K., M. Hedlin, C. de Groot-Hedlin, J. Vergoz, A. Le Pichon, and D. Drob, Source location of the 19 February 2008 Oregon bolide using seismic networks and infrasound arrays, J. Geophys. Res., 115, B12329, 2010 .

M. Yamada (e-mail: masumi@eqh.dpri.kyoto-u.ac.jp) and J. Mori 